\title{
A Two-Stage Fuzzy Logic Control Method of Traffic Signal Based on Traffic Urgency Degree
}

\author{
Yan Ge \\ School of Information Science and Technology, Qingdao University of Science and Technology, Qingdao 266061, China \\ Correspondence should be addressed to Yan Ge; geyanqd@gmail.com
}

Received 9 April 2014; Revised 10 July 2014; Accepted 11 July 2014; Published 4 August 2014

Academic Editor: Aiguo Song

Copyright (C) 2014 Yan Ge. This is an open access article distributed under the Creative Commons Attribution License, which permits unrestricted use, distribution, and reproduction in any medium, provided the original work is properly cited.

\begin{abstract}
City intersection traffic signal control is an important method to improve the efficiency of road network and alleviate traffic congestion. This paper researches traffic signal fuzzy control method on a single intersection. A two-stage traffic signal control method based on traffic urgency degree is proposed according to two-stage fuzzy inference on single intersection. At the first stage, calculate traffic urgency degree for all red phases using traffic urgency evaluation module and select the red light phase with large traffic urgency as the next phase to switch. At the second stage, green delay of the current green phase is determined by fuzzy inference based on the amount of vehicles of current green phase and next green phase. The average vehicle delays are used to evaluate the performance of the fuzzy signal controller. Finally, comparisons have been made with pretimed controller and fuzzy logic controller without considering the urgency of red phase. Simulation results show the performance of our proposed method.
\end{abstract}

\section{Introduction}

The monitoring and control of city traffic are becoming a major problem in many countries. With the ever increasing number of vehicles on the road, the traffic monitoring authority has to find new ways or measures for overcoming such a problem.

Traffic control in most signalized traffic intersections is done with either pretimed signal control or traffic-actuated control. Pretimed control is based on preset signal timings and therefore nonresponsive to real-time fluctuations in the traffic demand. Traffic-actuated control presents an improvement over pretimed control, but it has limited ability to respond to real-time traffic demand. For an intersection with actuated control, performance generally deteriorates with heavy traffic conditions and the proportion of stopped vehicles is generally high. Adaptive controllers are designed to address these deficiencies, as they have the ability to make real-time adjustments to signal settings in response to both observed and predicted real-time traffic demands.

Several approaches have been proposed for the design and implementation of adaptive signal control systems. The major research focus has been on application of fuzzy logic on intersection control. Fuzzy logic was initiated in 1968 by Zadeh [1]. Fuzzy logic is based on mathematical representation of human knowledge and experiences. It reasons approximately or in a certain degree of true or false. A fuzzy system is a classical artificial intelligent technology using fuzzy logic to solve problems in many fields. It has been applied successfully in many control systems, such as robot control, traffic signal management, industrial control, and home appliances, where traditional modeling techniques and controllers do not usually provide satisfactory system performance [2-6].

There are many earlier applications of fuzzy logic to traffic signal control. Pappis and Mamdani have used fuzzy logic to control isolated two-way intersection, with no turning vehicle movements [7]. Niittymäki and Turunen provided a fuzzy IFTHEN control algorithm based on Lukasiewicz equivalence to signalized isolated pedestrian crossing [8]. Kaedi et al. established a two-stage method for intersection signal timing control based on neurofuzzy network [9]. Mehan provided a fuzzy logic signal controller for a four-way isolated intersection. This model is suited for mixed traffic, including the high proportion of motorcycles [10]. Hou et al. established a fuzzy control model for traffic light with countdown ability. It implemented a self-adapted fuzzy controller for intersection signal control based on the conception of flow quantitative 


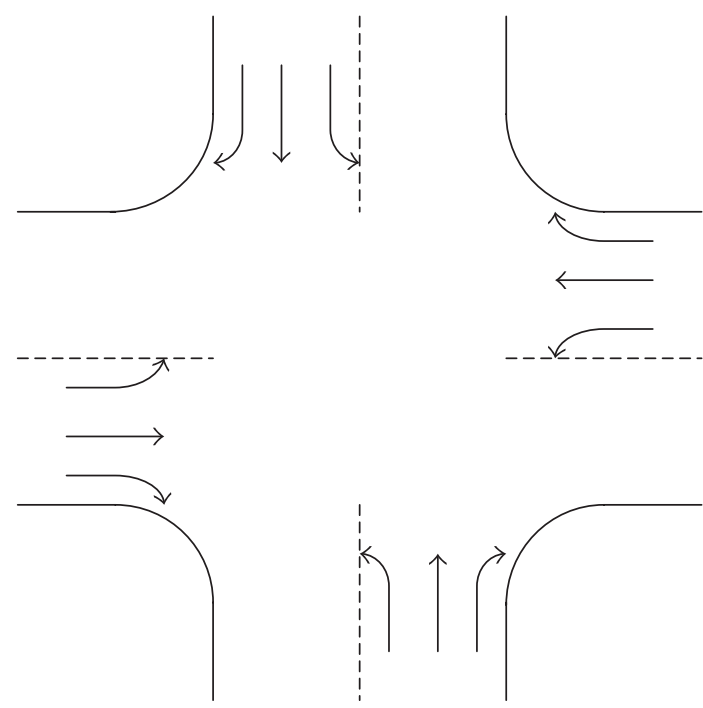

FIgURE 1: The typical intersection with four phases.

to fuzzy traffic flow [11]. Pranevicius and Kraujalis presented a traffic signal control method based on fuzzy logic for an isolated signalized intersection. The current green signal can be extended or terminated in response to changing traffic conditions [12].

All the researches reviewed above have reported better performance of the fuzzy logic controllers compared to pretimed and actuated controllers. However, the reviewed research switched traffic phase in sequence without considering the urgency of red phase. The main objective of this research is to design a fuzzy logic-based signal controller considering the urgency of red phase for a four-way isolated intersection with through and left-turning vehicle movements. The fuzzy logic-based signal controller will not only decide whether to extend or terminate a current green phase but also decide which red phase will be set as green phase. The average vehicle delays will be used to evaluate the performance of the fuzzy signal controller. The comparisons will be made with pretimed controller and fuzzy logic controller without considering the urgency of red phase.

\section{Fuzzy Logic Signal Control Method Based on Traffic Urgency Degree}

This paper researches an isolated four-way intersection with through and left-turning vehicle movements (Figure 1). The preset changing order of four phases is illustrated in Figure 2.

2.1. The Distribution of Vehicles Arrival. The distribution of vehicles arrival is a discrete random distribution, also known as the count distribution. It reflected a random vehicles number within a fixed period of time at a given spot. The common distributions of vehicle arrival are Poisson distribution and binomial distribution. In this paper, we assume that the arrival vehicles from all directions to an intersection are random and obey the Poisson distribution. The vehicle arrival rate is $0 \sim 0.4$ per second.

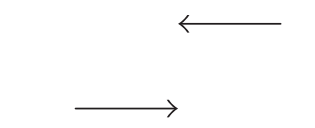

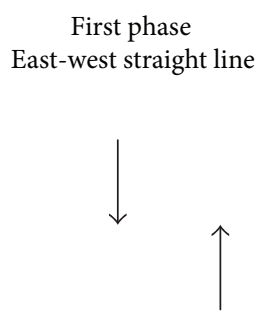

Third phase
South-north straight line

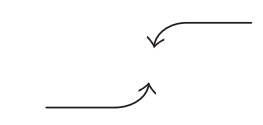

Second phase East-west left line

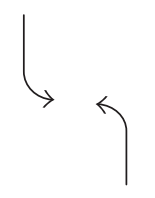

Fourth phase South-north left line
FIGURE 2: The four-phase order of an intersection.

The function of Poisson distribution is as follows [13]:

$$
P(x)=\frac{(\lambda t)^{x} e^{-\lambda t}}{x !}, \quad x=0,1,2, \ldots,
$$

where $P(x)$ is the probability of vehicles $x$ during counting interval $t$; $\lambda$ is the average arrival rate per unit time; $t$ is the duration of each counting interval.

Set $m=\lambda t$, where $m$ is the average number of vehicles during counting interval. The function (1) can be rewritten as follows:

$$
P(x)=\frac{m^{x} e^{-m}}{x !}
$$

2.2. The Average Delay of Vehicles. In this paper, the average delay of vehicles is the performance evaluation for traffic signal control of intersection. If the average vehicle delay is smaller, the traffic signal control effect is better. The calculation of vehicle delay model is shown below. The amount of queuing vehicles at time $t$ in red light phase $Q_{r t}$ can be calculated as follows:

$$
Q_{r t}=\sum_{i=1}^{k}\left(Q_{g i}+\sum_{j=1}^{t} q_{i j}\right),
$$

where $t$ is the traffic time; $r$ is a symbol representing the red phase; $Q_{r}$ is the amount of queuing vehicles in red light phase; $i$ is one of the red light phase branches; $j$ is the time interval during the red light; $g$ is a symbol representing the green phase; $Q_{g i}$ is the amount of queuing vehicles in the red light phase branch $i$, when the current green signal ends; $q_{i j}$ is the amount of arriving vehicles in the red light phase branch $i$ during time interval $j ; k$ is the number of red phase branch. $k=6$, ignoring the right turn traffic.

The total vehicle delay in red light phase can be calculated as follows:

$$
D_{r}=\sum_{t=1}^{n}\left[\sum_{i=1}^{k}\left(Q_{g i}+\sum_{j=1}^{t} q_{i j}\right)\right],
$$

where $n$ is the red light time of red light phase. 


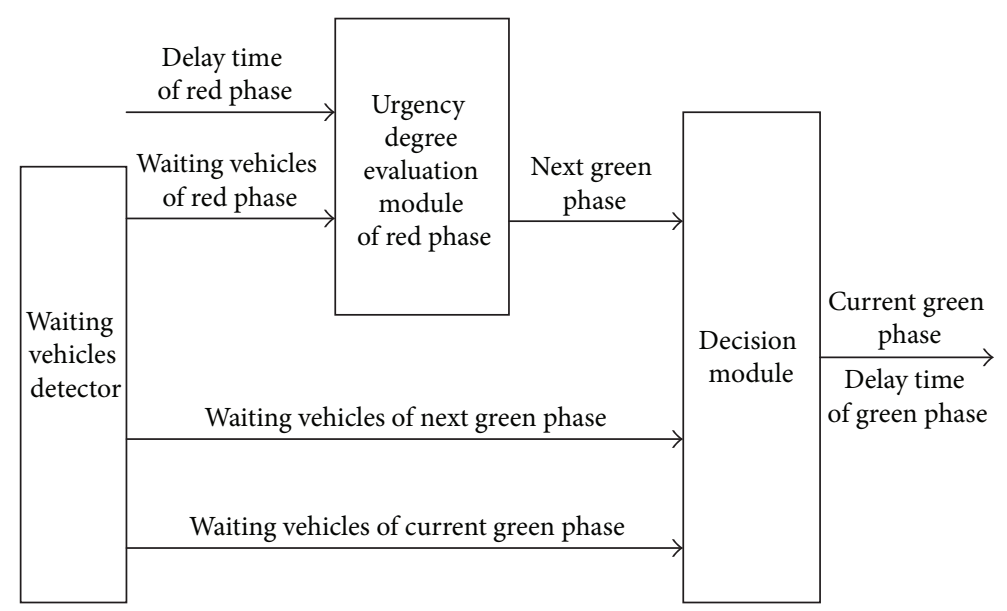

FIGURE 3: The chart of fuzzy control principle based on the urgency degree of red traffic phase.

The amount of queuing vehicles at the $t$ seconds during green light can be calculated as follows:

$$
Q_{g t}=\sum_{i=1}^{k} z_{i}\left(Q_{r i}+\sum_{j=1}^{t} q_{i j}-q_{s} \cdot t\right),
$$

where $q_{s}$ is saturation flow; $q_{i j}$ is the amount of arriving vehicles in the green light phase branch $i$ during time interval; $Q_{r i}$ is the amount of arriving vehicles in the green light phase branch $i$ during last red light phase; $k$ is the vehicles flow in green light phase. In this paper, there are four phases in intersection regardless of right turn flow. Consider that $k=2$. The value $z_{i}$ can show whether there are queuing vehicles or not in current green light phase branch $i$. If $Q_{r i}+\sum_{j=1}^{t} q_{i j}-$ $q_{s} \cdot t>0$, then $z_{i}=1$; otherwise, $z_{i}=0$.

The total vehicle delay of green light phase can be calculated as follows:

$$
D_{g}=\sum_{t=1}^{l}\left[\sum_{i=1}^{k} z_{i}\left(Q_{r i}+\sum_{j=1}^{t} q_{i j}-q_{s} \cdot t\right)\right],
$$

where $l$ is the green light time during green light phase.

The total vehicle delay in the $x$ cycle can be calculated as follows:

$$
D_{x}=D_{r}+D_{g}
$$

The average vehicle delay can be calculated as follows:

$$
d=\frac{\sum_{x=1}^{y} D_{x}}{A}
$$

where $y$ is the number of cycles; $A$ is the total amount of arriving vehicles from all directions in an intersection during all cycles.

\subsection{Fuzzy Logic Signal Control Principle Based on the Urgency} of Red Traffic Phase. In this paper, the fuzzy logic-based signal controller will not only decide whether to extend or terminate a current green phase but also decide which red phase will be set as green phase. That is, the phase sequence is uncertain. But each phase must be guaranteed one and only one time in a signal cycle. The fuzzy controller based on traffic urgency degree is shown in Figure 3.

The core control algorithms for fuzzy logic-based traffic signal include the following steps.

Step 1. Set a minimum green time for each phase according to the actual traffic condition.

Step 2. Set the minimum green time for the current green phase.

Step 3. Calculate traffic urgency degree for all red phases using traffic urgency evaluation module.

Step 4. Select the red light phase with large traffic urgency as the next phase to switch.

Step 5. Get the current green phase green light time delay through fuzzy reasoning based on the vehicles number of the current green phase and the next green phase.

Step 6. Switch to the next green phase; skip to Step 2 and continue.

\section{Design of Fuzzy Logic-Based Signal Controller Based on Traffic Urgency Degree}

Fuzzy logic-based signal controller based on traffic urgency includes the following two modules.

(i) The traffic urgency degree evaluation module for red light phase: calculate traffic urgency degree for all of the red phase using the traffic urgency evaluation module. Select the red light phase with large traffic urgency as the next phase to switch.

(ii) Decision module: get the current green phase green light time delay through fuzzy reasoning according to the vehicles number of the current green phase and the next green phase. 


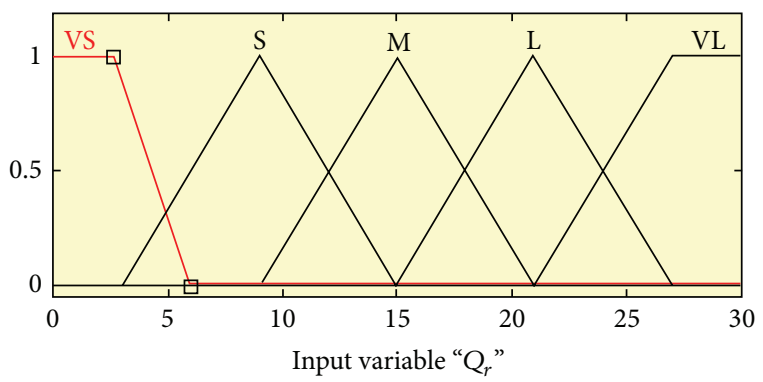

Figure 4: The membership function of input variable $Q_{r}$.

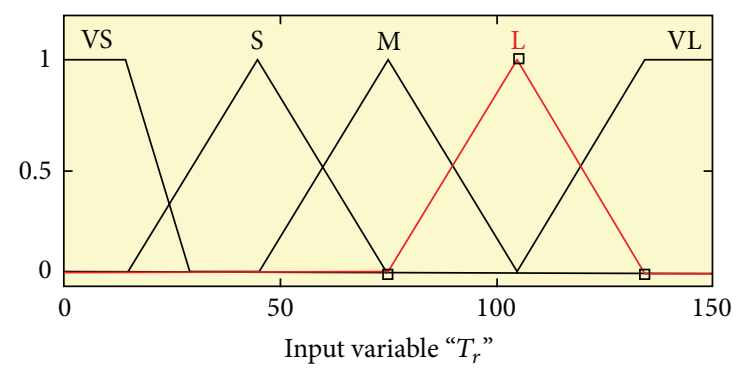

FIGURE 5: The membership function of input variable $T_{r}$.

3.1. Traffic Urgency Degree Evaluation Module for Red Light Phase. There are two steps in the traffic urgency degree evaluation module for red light phase. Calculate traffic urgency degree for all of the red phase using the traffic urgency evaluation module. Select the red light phase with large traffic urgency as the next phase to switch.

There are two input variables and one output variable for traffic urgency degree evaluation module. The input variables include vehicles number of current red light phase $Q_{r}$ and the duration of red light since the last end of the green light $T_{r}$. The output variable is traffic urgency of all current red light phase $U_{r}$. The membership functions of $Q_{r}, T_{r}$, and $U_{r}$ are defined as follows.

The domain of $Q_{r}$ is $[0,30]$. It is divided into five fuzzy subsets \{Very Short, Short, Medium, Long, Very Long\}, for short $\{\mathrm{VS}, \mathrm{S}, \mathrm{M}, \mathrm{L}, \mathrm{VL}\}$. The membership function of $Q_{r}$ is shown in Figure 4. The domain of $T_{r}$ is $[0,150]$. It is divided into five fuzzy subsets \{Very Short, Short, Medium, Long, Very Long\}, for short $\{\mathrm{VS}, \mathrm{S}, \mathrm{M}, \mathrm{L}, \mathrm{VL}\}$. The membership function of $T_{r}$ is shown in Figure 5. The domain of $U_{r}$ is $[0,1]$. It is divided into five fuzzy subsets \{Very Small, Small, Medium, Big, Very Big\}, for short $\{\mathrm{VS}, \mathrm{S}, \mathrm{M}, \mathrm{B}, \mathrm{VB}\}$. The membership function of $U_{r}$ is shown in Figure 6.

The fuzzy rules of red light phase urgency degree evaluation module are shown in Table 1. In Table 1, the element of the first row and first column is "VS," which represents a fuzzy rule. The sample rule is as follows:

$$
\text { if }\left\{Q_{r} \text { is VS }\right\} \text { and }\left\{T_{r} \text { is VS }\right\} \text { then }\left\{U_{r} \text { is VS }\right\} \text {. }
$$

This fuzzy rule can be described by natural languages as follows: "If the queue number of current red light phase is very short and the light duration of current red light phase is
TABLE 1: The fuzzy rules of evaluation module for red light phase urgency degree.

\begin{tabular}{lccccc}
\hline$Q_{r}$ & & & $T_{r}$ & & \\
& VS & S & M & L & VL \\
\hline VS & VS & VS & S & S & M \\
S & VS & S & S & M & M \\
M & M & S & M & M & B \\
L & B & M & M & B & VB \\
VL & B & M & B & VB & VB \\
\hline
\end{tabular}

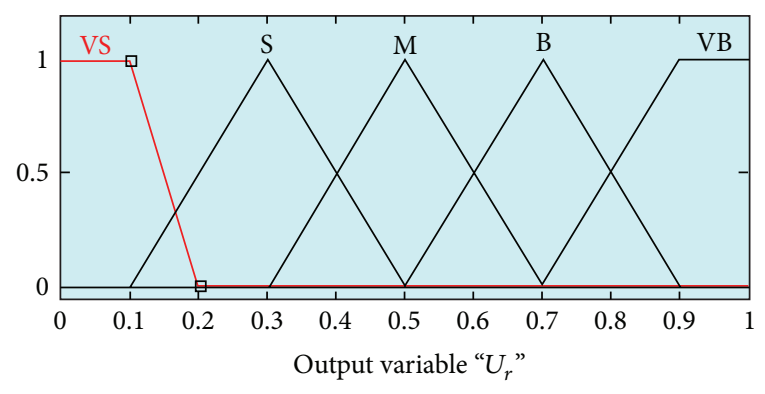

FIGURE 6: The membership function of output variable $U_{r}$.

very short, so the urgency degree of current red light phase is very small."

\subsection{Decision Module for Delay Time of the Current Green Light}

Phase. In decision module, the current green phase green light time delay can be acquired according to the vehicles number of the current green phase and the next green phase. There are two input variables and one output variable for the decision module. The input variables include $Q_{g}$ and $Q_{r} \cdot Q_{g}$ is the vehicles number of current green light phase, and $Q_{r}$ is vehicles number of red light phase which has big traffic urgency. The output variable is the delay time of current green light phase $G_{e}$.

The domain of $Q_{g}$ is $[0,20]$. It is divided into five fuzzy subsets \{Very Short, Short, Medium, Long, Very Long\}, for short $\{\mathrm{VS}, \mathrm{S}, \mathrm{M}, \mathrm{L}, \mathrm{VL}\}$. The domain of $Q_{r}$ is $[0,30]$. It is divided into five fuzzy subsets \{Very Short, Short, Medium, Long, Very Long\}, for short $\{\mathrm{VS}, \mathrm{S}, \mathrm{M}, \mathrm{L}, \mathrm{VL}\}$. The domain of $G_{e}$ is $[0,30]$. It is divided into five fuzzy subsets \{Very Short, Short, Medium, Long, Very Long\}, for short \{VS, S, M, L, $\mathrm{VL}$. The fuzzy rules of decision module for the delay time of current green light phase are shown in Table 2. In Table 2, the element of the first row and second column is "VS," which represents a fuzzy rule. The sample rule is as follows:

$$
\text { if }\left\{Q_{r} \text { is } S\right\} \text { and }\left\{Q_{g} T_{r} \text { is VS }\right\} \text { then }\left\{G_{e} \text { is VS }\right\} \text {. }
$$

This fuzzy rule can be described by natural languages as follows: "If the queue number of current green light phase is very short and the queue number of next green light phase is short, so the delay time of current green light phase is very small." 
TABLE 2: The fuzzy rules of decision module for the delay time of current green light phase.

\begin{tabular}{|c|c|c|c|c|c|}
\hline \multirow{2}{*}{$Q_{g}$} & \multicolumn{5}{|c|}{$Q_{r}$} \\
\hline & VS & $\mathrm{S}$ & $\mathrm{M}$ & $\mathrm{L}$ & VL \\
\hline VS & VS & VS & VS & VS & VS \\
\hline S & $S$ & $S$ & $S$ & VS & VS \\
\hline $\mathrm{M}$ & $\mathrm{M}$ & $\mathrm{M}$ & $\mathrm{M}$ & $\mathrm{M}$ & $S$ \\
\hline $\mathrm{L}$ & $\mathrm{L}$ & $\mathrm{L}$ & $\mathrm{L}$ & $\mathrm{M}$ & $\mathrm{M}$ \\
\hline $\mathrm{VL}$ & VL & VL & $\mathrm{L}$ & $\mathrm{L}$ & $\mathrm{L}$ \\
\hline
\end{tabular}

TABLE 3: Average vehicle delays of low traffic flow.

\begin{tabular}{lccc}
\hline Number of simulation & Pretimed control & Fuzzy control & Fuzzy control with traffic urgency degree \\
\hline 1 & 73.5387 & 45.6423 & 40.7576 \\
2 & 85.3338 & 42.7256 & 37.5516 \\
3 & 82.7172 & 42.1008 & 42.0751 \\
4 & 79.6795 & 44.7148 & 43.9731 \\
5 & 91.9941 & 41.3830 & 40.4205 \\
Average delay & $\mathbf{8 2 . 6 5 2 6 6}$ & $\mathbf{4 3 . 3 1 3 3}$ & $\mathbf{4 0 . 9 5 5 5 8}$ \\
\hline
\end{tabular}

TABLE 4: Average vehicle delays of middle traffic flow.

\begin{tabular}{lccc}
\hline Number of simulation & Pretimed control & Fuzzy control & Fuzzy control with traffic urgency degree \\
\hline 1 & 146.6501 & 82.4300 & 76.7970 \\
2 & 108.8296 & 63.1138 & 59.2166 \\
3 & 187.9133 & 82.8549 & 78.5025 \\
4 & 150.2061 & 78.3128 & 71.8656 \\
5 & 175.1695 & 86.8280 & 74.6925 \\
Average delay & $\mathbf{1 5 3 . 7 5 3 7}$ & $\mathbf{7 8 . 7 0 7 9}$ & $\mathbf{7 2 . 2 1 4 8 4}$ \\
\hline
\end{tabular}

\section{Simulation Analysis}

The average vehicle delays were used to evaluate the performance of the fuzzy signal controller. The comparisons have been made with pretimed controller and fuzzy logic controller without considering the urgency of red phase.

In order to make comparisons, identical conditions have to be set during the simulations. The minimum green time and the delay time were set as follows.

(i) The minimum green time of east-west straight phase and north-south straight phase was set as 20 seconds.

(ii) The minimum green time of east-west left turn phase was set as 15 seconds.

(iii) The maximum delay of green light for green light phase was set as 30 seconds.

(iv) If the vehicle arriving in the intersection is stochastic and the traffic flow is not large, the vehicle arrival obeys the Poisson distribution. So, the vehicle arrival rate in the intersection was set as $0 \sim 0.4$ per second.

(v) Assume that the vehicle leaving rate is one car leaving waiting team per second, when a phase from red to green light.

(vi) Set the simulation time as 1 hour.
In this paper, the vehicle arrival rate is divided into three types, including low traffic flow, middle traffic flow, and high traffic flow. The ranges of each stage vehicle rate are as follows: $0 \sim 0.15$ car per second, $0.15 \sim 0.3$ car per second, and $0.3 \sim$ 0.4 car per second. The average delay of pretimed control, fuzzy control, and fuzzy control with traffic urgency degree is shown in Table 3 (simulation results of low traffic flow), Table 4 (simulation results of middle traffic flow), and Table 5 (simulation results of high traffic flow).

From Tables 3, 4, and 5, we can analyze the average delay of pretimed control, fuzzy control, and fuzzy control with traffic urgency degree, in three traffic types. The simulation results are as follows.

(i) In the low traffic flow, the average vehicle delay of fuzzy control with traffic urgency degree is reduced by $45.6 \%$ compared to pretimed control and is deduced by $5.4 \%$ compared to fuzzy control.

(ii) In the middle traffic flow, the average vehicle delay of fuzzy control with traffic urgency degree is reduced by $48.8 \%$ compared to pretimed control and is deduced by $8.2 \%$ compared to fuzzy control.

(iii) In the high traffic flow, the average vehicle delay of fuzzy control with traffic urgency degree is reduced by $30.6 \%$ compared to pretimed control and is deduced by $2.36 \%$ compared to fuzzy control. 
TABle 5: Average vehicle delays of high traffic flow.

\begin{tabular}{lccc}
\hline Number of simulation & Pretimed control & Fuzzy control & Fuzzy control with traffic urgency degree \\
\hline 1 & 304.7168 & 211.6806 & 208.7081 \\
2 & 314.6766 & 213.7743 & 209.5257 \\
3 & 308.4338 & 219.8659 & 206.8460 \\
4 & 310.3295 & 210.5916 & 209.1864 \\
5 & 311.6645 & 219.8842 & 216.4840 \\
Average delay & $\mathbf{3 0 9 . 9 6 4 2}$ & $\mathbf{2 1 5 . 1 5 9 3}$ & $\mathbf{2 1 0 . 1 5}$ \\
\hline
\end{tabular}

From the contrast of simulation results, the control effect of fuzzy control for traffic urgency degree is better than the pretimed control and common fuzzy control. The order of green light phase can be adjusted for some special traffic flow in fuzzy traffic control with traffic urgency degree.

\section{Conclusions}

This paper has analyzed the deficiency of existing fuzzy controller and put forward the strategies of improving fuzzy control based on traffic urgency degree. First, in order to determine the max urgency degree of red phase, the traffic urgency degrees of red phases were evaluated during the current green phase. The green light delay of current green phase was determined by fuzzy reasoning according to the number of vehicles of the current green phase and the next green phase. This algorithm has considered traffic factors and has more objectively reflected the intersection of each phase traffic demand urgent degree. Finally, the comparisons have been made with pretimed controller and fuzzy logic controller and fuzzy traffic control considering the urgency of red phase. From the contrast of the simulation results, the performance of fuzzy control for traffic urgency degree is better than the pretimed control and common fuzzy control method.

\section{Conflict of Interests}

The author declares that there is no conflict of interests regarding the publication of this paper.

\section{Acknowledgments}

This work was supported by the National Natural Science Foundation of China (Grant no. 61273180), the Natural Science Foundation of Shandong Province, China (Grant nos. ZR2009GQ013, ZR2011FQ005, and ZR2011FL010), the Project of Shandong Province Higher Educational Science and Technology Program, China (J14LN74), and the International Cooperation Program for Excellent Lecturers of 2012 by Shandong Provincial Education Department, China.

\section{References}

[1] L. A. Zadeh, "Fuzzy algorithms," Information and Control, vol. 12, no. 2, pp. $94-102,1968$.
[2] Z. Bingül and O. Karahan, "A Fuzzy Logic Controller tuned with PSO for 2 DOF robot trajectory control," Expert Systems with Applications, vol. 38, no. 1, pp. 1017-1031, 2011.

[3] P. G. Balaji and D. Srinivasan, "Type-2 fuzzy logic based urban traffic management," Engineering Applications of Artificial Intelligence, vol. 24, no. 1, pp. 12-22, 2011.

[4] R. Precup and H. Hellendoorn, "A survey on industrial applications of fuzzy control," Computers in Industry, vol. 62, no. 3, pp. 213-226, 2011.

[5] H. Singh, M. M. Gupta, T. Meitzler et al., "Real-life applications of fuzzy logic," Advances in Fuzzy Systems, vol. 2013, Article ID 581879, 3 pages, 2013.

[6] S. Jingzhuo and L. Lin, "Steady-state fuzzy modeling of ultrasonic motor system," Modelling and Simulation in Engineering, vol. 2011, Article ID 275158, 6 pages, 2011.

[7] C. P. Pappis and E. H. Mamdani, "A fuzzy logic controller for a traffic junction," IEEE Transactions on Systems, Man and Cybernetics, vol. 7, no. 10, pp. 707-717, 1977.

[8] J. Niittymäki and E. Turunen, "Traffic signal control on similarity logic reasoning," Fuzzy Sets and Systems, vol. 133, no. 1, pp. 109-131, 2003.

[9] M. Kaedi, N. Movahhedinia, and K. Jamshidi, “Traffic signal timing using two-dimensional correlation, neuro-fuzzy and queuing based neural networks," Neural Computing and Applications, vol. 17, no. 2, pp. 193-200, 2008.

[10] S. Mehan, "Introduction of traffic light controller with fuzzy control system," International Journal of Electronics \& Communication Technology, vol. 2, no. 3, pp. 119-122, 2011.

[11] R. Hou, Q. Wang, J. Wang, J. Wang, Y. Lu, and J. Kim, "A fuzzy control method of traffic light with countdown ability," International Journal of Control and Automation, vol. 5, no. 4, pp. 93-102, 2012.

[12] H. Pranevicius and T. Kraujalis, "Knowledge based traffic signal control model for signalized intersection," Transport, vol. 27, no. 3, pp. 263-267, 2012.

[13] M. Hosamo, "A study of the source traffic generator using poisson distribution for ABR service," Modelling and Simulation in Engineering, vol. 2012, Article ID 408395, 6 pages, 2012. 

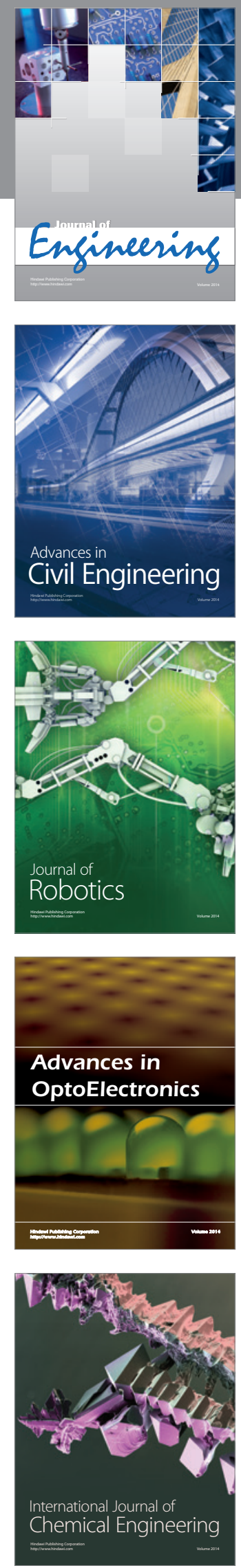

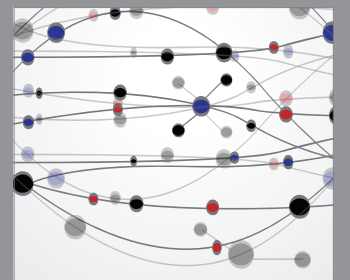

The Scientific World Journal
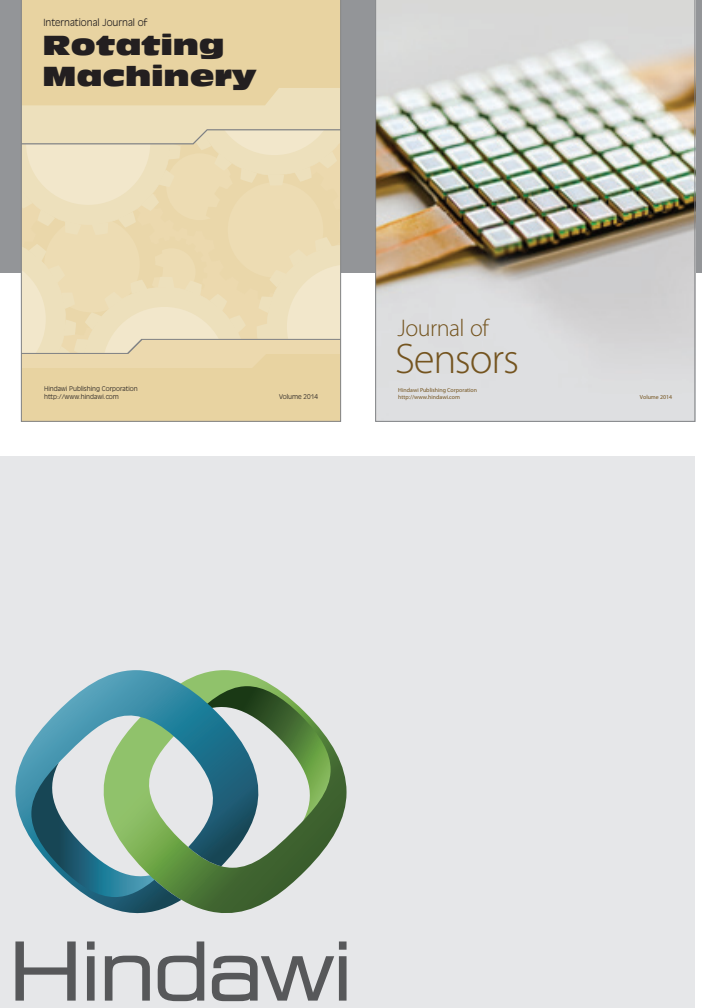

Submit your manuscripts at http://www.hindawi.com
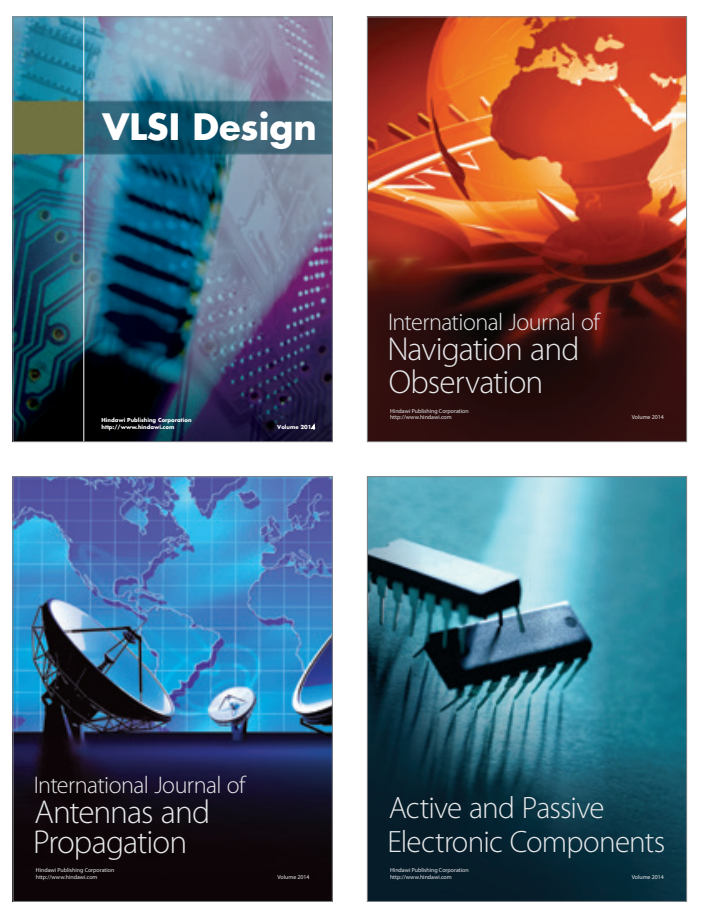
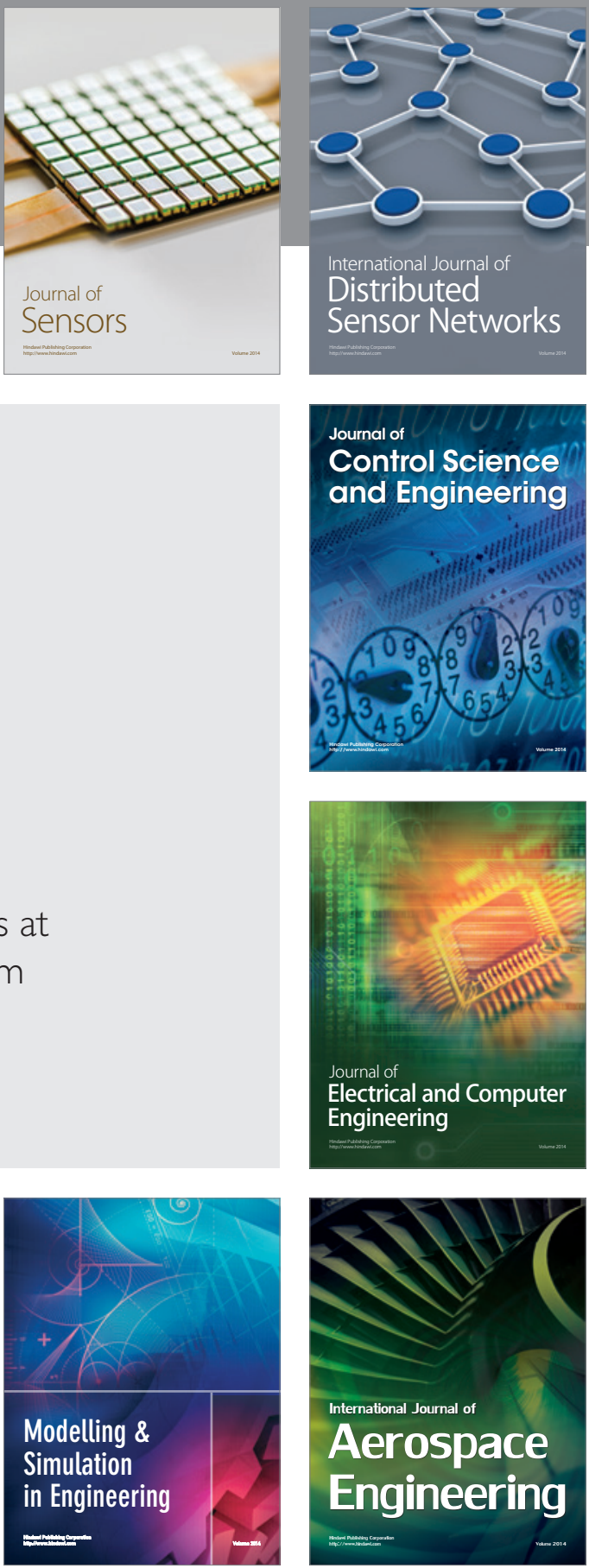

Journal of

Control Science

and Engineering
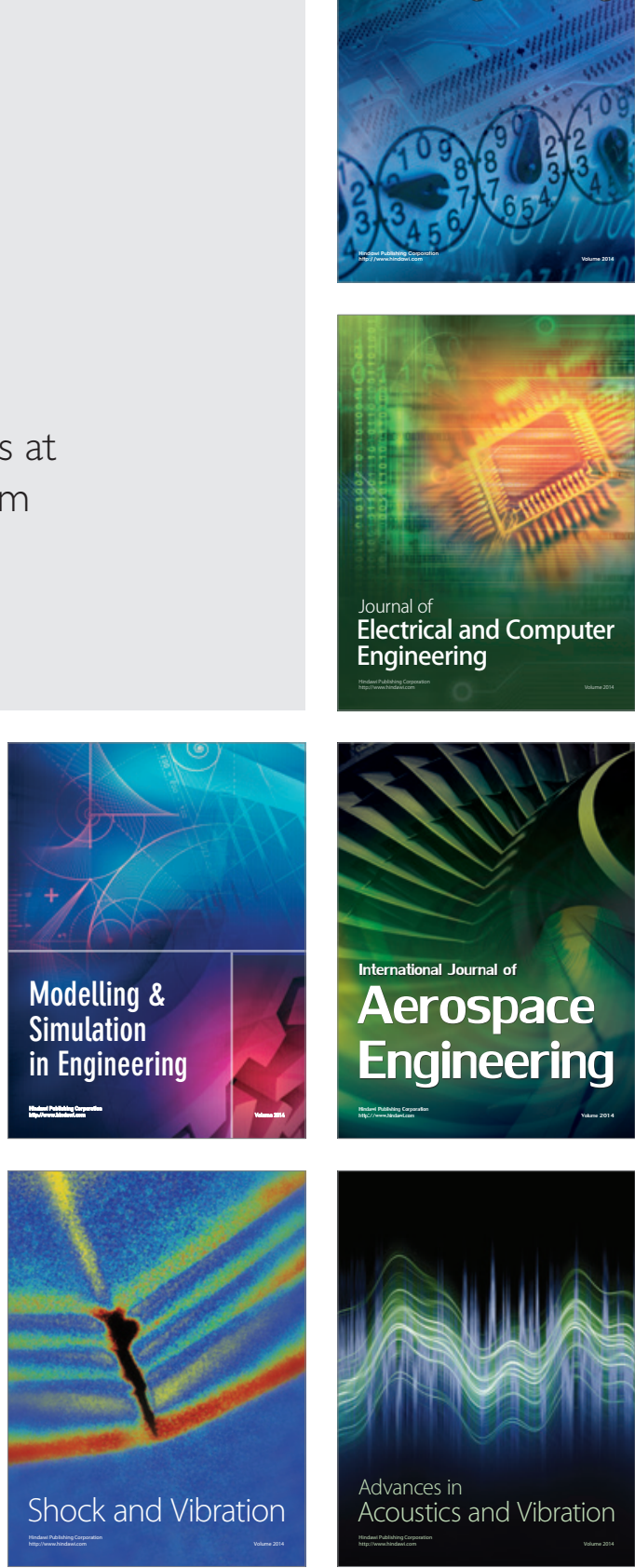